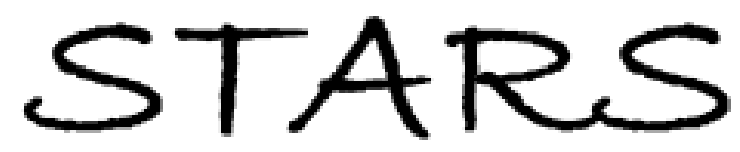

University of Central Florida

STARS

$1-1-1999$

\title{
Ensemble density-functional approach to charge-spin textures in inhomogeneous quantum Hall systems
}

O. Heinonen

University of Central Florida

J. M. Kinaret

M. D. Johnson

University of Central Florida

Find similar works at: https://stars.library.ucf.edu/facultybib1990

University of Central Florida Libraries http://library.ucf.edu

This Article is brought to you for free and open access by the Faculty Bibliography at STARS. It has been accepted for inclusion in Faculty Bibliography 1990s by an authorized administrator of STARS. For more information, please contact STARS@ucf.edu.

\section{Recommended Citation}

Heinonen, O.; Kinaret, J. M.; and Johnson, M. D., "Ensemble density-functional approach to charge-spin textures in inhomogeneous quantum Hall systems" (1999). Faculty Bibliography 1990s. 2672.

https://stars.library.ucf.edu/facultybib1990/2672

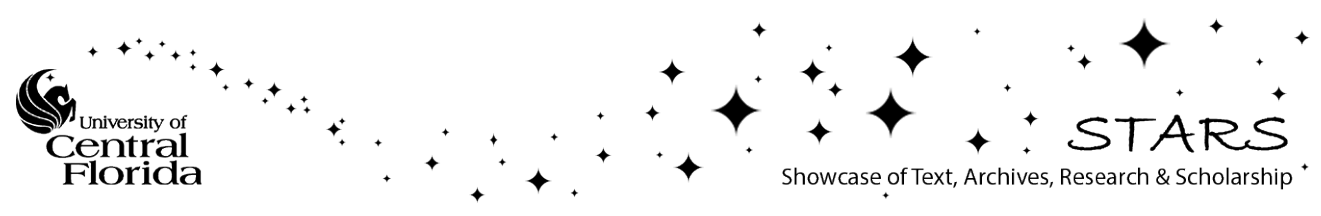




\title{
Ensemble density-functional approach to charge-spin textures in inhomogeneous quantum Hall systems
}

\author{
O. Heinonen* \\ Department of Physics, University of Central Florida, Florida 32816-2385 \\ J. M. Kinaret \\ Department of Applied Physics, Göteborg University, S-412 96 Göteborg, Sweden \\ and Chalmers University of Technology, S-412 96 Göteborg, Sweden \\ M. D. Johnson \\ Department of Physics, University of Central Florida, Florida 32816-2385 \\ (Received 11 February 1998; revised manuscript received 7 October 1998)
}

\begin{abstract}
We extend our ensemble density-functional approach to quantum Hall systems to include noncollinear spins to study charge-spin textures in inhomogeneous quantum Hall systems. We have studied the edge reconstruction in quantum dots at unit bulk filling factor and at $1 / 3$ bulk filling factor as a function of the stiffness of an external confining potential. For soft enough edges, these systems reconstruct to a state in which the electron spins rotate gently as the edge is approached, with a nontrivial spin-charge texture at the edge of the system. [S0163-1829(99)11411-5]
\end{abstract}

\section{INTRODUCTION}

It has recently become clear that the spin degree of freedom plays a significant role near ferromagnetic fillings in the quantum Hall effect (QHE). ${ }^{1-3}$ This is because of the low ratio of the Zeeman energy $E_{Z}=g^{*} \mu_{B} B$ to the Coulomb energy $E_{C}=e^{2} /\left(\epsilon_{0} l_{B}\right)$. Here, $g^{*}$ is the Landé $g$ factor, $\mu_{B}$ the Bohr magneton, $B$ the applied magnetic field strength, $\epsilon_{0}$ the static dielectric constant, and $l_{B}=\sqrt{\hbar c / e B}$ the magnetic length. For GaAs systems, the low values of the Landé factor and of the electron effective mass $m^{*}$ conspire to make $\tilde{g}$ $=E_{Z} / E_{C} \approx E_{Z} /\left(\hbar \omega_{c}\right) \lesssim 0.02$ for fields in the range of a few to approximately $10 \mathrm{~T}$. Here, $\omega_{c}=e B /\left(m^{*} c\right)$ is the cyclotron frequency. Nonetheless, single-particle spin-flip excitations still cost a large amount of energy, because of the loss of exchange energy associated with a spin flip. This means that the spin degree of freedom is controlled by the interelectron Coulomb energy, and not by the Zeeman energy. One consequence is that if a single spin is reversed, it becomes energetically favorable for the system to smoothly rotate the magnetization direction to restore it over some distance from the reversed spin. Due to the connection between flux and charge density in incompressible ferromagnetic QHE ground states, such spin textures also acquire a charge density, and the resulting spin-charge textures are commonly called "skyrmions." (More accurately, skyrmions are the particular type of spin-charge textures that show up in certain continuum models, such as the nonlinear $\sigma$ model ${ }^{4}$ ) There is now ample theoretical ${ }^{2,5,6}$ and experimental work ${ }^{7-9}$ suggesting that such skyrmions are indeed the low-energy charged excitations, at least near filling factor $\nu=1$. This is, for example, manifested in the rapid destruction of the groundstate polarization observed experimentally ${ }^{7}$ as the filling factor is varied away from unity. Recent theoretical work ${ }^{10-13}$ has also indicated that the edge reconstruction of ferromagnetic QHE systems may acquire nontrivial spin textures (or charge-density waves) as the edge confinement is softened.
This may radically alter our understanding of edges in QHE systems, and, concomitantly, our interpretations of experiments that probe the gapless edge modes. This is at the present best understood for edges of $\nu=1$ systems. One question we wish to address here is whether similar chargespin textures can occur at the edges of systems in regimes of the ferromagnetic fractional quantum Hall effect (FQHE) (such as $\nu=1 / 3$ ), where not just electron exchange but also correlations are important.

We have previously developed an ensemble densityfunctional approach for spin-polarized systems, ${ }^{14,15}$ and subsequently generalized that approach to include the electron spin, but with the spin quantization axis constrained to be parallel to the external magnetic field. ${ }^{16}$ We present here a further extension that is able to deal with a rotating spin quantization axis. Advantages of our DFT approach are that it includes electron interactions beyond exchange, and that it can be applied to large inhomogeneous fractional QHE systems. This makes our ensemble spin DFT approach the only available method that can be applied to general inhomogeneous QHE systems, spanning regimes from the FQHE to the semiclassical, and which includes the spin degree of freedom and Landau-level mixing. We have used this approach to study the edge reconstruction of circularly symmetric quantum dots. Our results show that as the edge confinement is softened, the system goes from a spin-polarized sharp edge to a softer edge with a nontrivial spin texture, in agreement with results obtained by other groups using the Hartree-Fock approximation (at integer filling) or field-theoretical models. ${ }^{10}$ A new result here is to show how the reconstruction to a spin-charge textured edge can also happen for FQHE systems.

This paper is organized as follows. In Sec. II, we present the general ensemble density-functional theory for noncollinear spins. Section III presents some technical details of the theory, including an extension of previously used exchangecorrelation energies for QHE systems to include both higher Landau levels and electron spin. In Sec. IV we present re- 
sults from numerical calculations of quantum dots at unit bulk filling factor and at $1 / 3$ bulk filling factor. Finally, Sec. $\mathrm{V}$ contains conclusions and a discussion.

\section{ENSEMBLE SPIN-DENSITY-FUNCTIONAL THEORY FOR NONCOLLINEAR SPINS}

In its most general formulation, spin-density-functional theory, as developed by von Barth and Hedin, ${ }^{17}$ allows for noncollinear spins. This is based on a generalization of the Hohenberg-Kohn ${ }^{18}$ theorem in which the electron density $n(\mathbf{r})$ is replaced by the single-particle density matrix $\rho_{\sigma, \sigma^{\prime}}(\mathbf{r}) \equiv\left\langle 0\left|\hat{\psi}_{\sigma}^{\dagger}(\mathbf{r}) \hat{\psi}_{\sigma^{\prime}}(\mathbf{r})\right| 0\right\rangle$, where $\hat{\psi}_{\sigma}(\mathbf{r}) \quad\left(\hat{\psi}_{\sigma}^{\dagger}(\mathbf{r})\right)$ is the usual electron annihilation (creation) operator for an electron of spin $\sigma$ at position $\mathbf{r}$, and $|0\rangle$ is the ground state. We will use notations in which $\sigma= \pm 1$ or $\sigma=\uparrow, \downarrow$, with up spin ( $\uparrow$ ) corresponding to $\sigma=-1$. A computationally useful approach is then constructed in the usual way by considering an auxiliary noninteracting system in some effective external potential $v_{s}(\mathbf{r})$ chosen so that this system has the same groundstate single-particle density matrix $\rho_{\sigma, \sigma^{\prime}}(\mathbf{r})$ as the interacting system at hand. A variational principle associated with the generalized Hohenberg-Kohn theorem then yields KohnSham (KS) equations, ${ }^{19}$ which now include spin-dependent exchange-correlation potentials

$$
V_{\mathrm{xc}, \sigma \sigma^{\prime}}(\mathbf{r}) \equiv \frac{\delta E_{\mathrm{xc}}\left[\rho_{\sigma, \sigma^{\prime}}(\mathbf{r})\right]}{\delta \rho_{\sigma, \sigma^{\prime}}(\mathbf{r})} .
$$

A difficulty is that one does not usually have reliable approximations for the exchange-correlation potentials $V_{\mathrm{xc}, \sigma \sigma^{\prime}}(\mathbf{r})$, not even in the local-density approximation (LDA). Consequently, the density matrix is usually assumed to be diagonal for all $\mathbf{r}$, which means that the direction of the magnetization is assumed to be constant, and that direction is then conveniently chosen as the spin quantization axis. Thus, only up- and down-spin densities enter into the KS equations, and for the LDA (or extensions including generalized gradient approximations), one only needs to know the exchange-correlation energy $E_{\mathrm{xc}}(n, \xi)$ of a uniform system of density $n=n_{\uparrow}+n_{\downarrow}$ and polarization $\xi \equiv\left(n_{\uparrow}+n_{\downarrow}\right) /\left(n_{\uparrow}\right.$ $\left.-n_{\downarrow}\right)$. There exist now very accurate calculations of $E_{\mathrm{xc}}(n, \xi)$ for two- and three-dimensional electron gases (in zero magnetic field). ${ }^{20,21}$

However, the approximation of constant magnetization direction obviously does not work in systems for which it is known that the magnetization direction changes in space. Examples of such systems are $\mathrm{Mn}_{3} \mathrm{Sn},{ }^{22} \gamma$-Fe, ${ }^{23} \mathrm{U}_{3} \mathrm{Pt}_{4},{ }^{24}$ and QHE systems near unit filling. For such systems the full single-particle density matrix has to be used, and the problem then arises as to how one should construct a LDA. We will here follow an approach developed by Kübler et al. ${ }^{25}$ Sticht, Höck, and Kübler, ${ }^{22}$ and Sandratskii, ${ }^{26}$ and extend their approach to an ensemble DFT appropriate for QHE systems. The basic idea is to locally rotate the spin quantization axis to obtain a representation that locally diagonalizes the single-particle density matrix. The advantage of this procedure is that in order to construct a LDA, one then only needs the exchange-correlation energy as a function of spin-up and spin-down densities (or total density and polarization), for which approximations exist. The price one has to pay is to introduce local spin rotation angles $\theta(\mathbf{r})$ and $\varphi(\mathbf{r})$, which complicates the KS equations. This approach has given good results in applications to materials such as $\gamma$-Fe, ${ }^{23} \mathrm{U}_{3} \mathrm{Pt}_{4},{ }^{24}$ and $\alpha$ - $\mathrm{Fe}_{2} \mathrm{O}_{3} .{ }^{27}$ It can also give the spin stiffness important in studying spin-charge textures in the QHE. That this can happen in the LDA is not immediately obvious-in field-theoretical approaches the spin stiffness enters via a gradient of magnetization, and such gradients are neglected in the LDA. We will show in Sec. III that the LDA does give a spin stiffness, although formally its origin appears a bit different.

We now proceed to review the LDA approach of Kübler et $a l^{25}$ Sticht, Höck, and Kübler. ${ }^{22}$ We begin by writing the ground-state energy as a functional of the single-particle density matrix for a two-dimensional system in a constant external magnetic field $\mathbf{B}=B \hat{\mathbf{z}}$ :

$$
\begin{aligned}
E\left[\rho_{\sigma, \sigma^{\prime}}(\mathbf{r})\right]= & T_{s}\left[\rho_{\sigma, \sigma^{\prime}}(\mathbf{r})\right] \\
& +\int n(\vec{r}) \nu_{\mathrm{ext}}(\vec{r}) d^{3} r \\
& +g^{*} \mu_{B} B \sum_{\sigma, \sigma^{\prime}} \sigma \delta_{\sigma, \sigma^{\prime}} \int \rho_{\sigma, \sigma^{\prime}}(\mathbf{r}) d^{2} r \\
& +\frac{1}{2} \iint n(\mathbf{r}) v_{H}\left(\mathbf{r}-\mathbf{r}^{\prime}\right) n\left(\mathbf{r}^{\prime}\right) d^{2} r d^{2} r^{\prime} \\
& +E_{\mathrm{xc}}\left[\rho_{\sigma, \sigma^{\prime}}(\mathbf{r})\right] .
\end{aligned}
$$

Here, $T_{s}\left[\rho_{\sigma, \sigma^{\prime}}(\mathbf{r})\right]$ is the kinetic energy functional of noninteracting electrons, which in our case includes the external magnetic field B. The particle density is $n(\mathbf{r})$ $=\operatorname{Tr} \rho_{\sigma, \sigma^{\prime}}(\mathbf{r}), v_{H}(\mathbf{r})$ is the Hartree potential, and $E_{\mathrm{xc}}\left[\rho_{\sigma, \sigma^{\prime}}(\mathbf{r})\right]$ is the exchange-correlation energy, which depends parametrically on the magnetic field (for ease of notation we omit this parametric dependence). Using the variational principle of the Hohenberg-Kohn theorem, Eq. (2) leads to Kohn-Sham equations of the form

$$
\begin{aligned}
& {\left[T_{\mathrm{s}}(\mathbf{r}) \hat{\mathbf{I}}+V_{H}(\mathbf{r}) \hat{\mathbf{I}}+\nu_{\mathrm{ext}}(\mathbf{r}) \hat{\mathbf{I}}+g \mu_{B} B\left(\begin{array}{cc}
1 & 0 \\
0 & -1
\end{array}\right)\right.} \\
& \left.\quad+\left(\begin{array}{cc}
V_{\mathrm{xc}, \uparrow \uparrow}(\mathbf{r}) & V_{\mathrm{xc}, \uparrow \downarrow}(\mathbf{r}) \\
V_{\mathrm{xc}, \downarrow \uparrow}(\mathbf{r}) & V_{\mathrm{xc}, \downarrow \downarrow}(\mathbf{r})
\end{array}\right)\right]\left(\begin{array}{l}
\psi_{i, \uparrow}(\mathbf{r}) \\
\psi_{i, \downarrow}(\mathbf{r})
\end{array}\right)=\epsilon_{i}\left(\begin{array}{l}
\psi_{i, \uparrow}(\mathbf{r}) \\
\psi_{i, \downarrow}(\mathbf{r})
\end{array}\right)
\end{aligned}
$$

for single-particle spinors $\psi_{i}=\left(\psi_{i, \uparrow}, \psi_{i, \downarrow}\right)$ (here, $\hat{\mathbf{I}}$ is the $2 \times 2$ identity matrix). The problem with Eq. (3) is the spindependent exchange-correlation potential $V_{\mathrm{xc}, \alpha \beta}(\mathbf{r})$. The approach taken by Kübler and co-workers is to locally find a representation in which the single-particle density matrix is diagonal with diagonal elements $n_{\uparrow}(\mathbf{r})$ and $n_{\downarrow}(\mathbf{r})$. We can then just use the chain rule and write

$$
V_{\mathrm{xc}, \alpha \beta}=\frac{\delta E_{\mathrm{xc}}\left[n_{\uparrow}, n_{\downarrow}\right]}{\delta n_{\uparrow}} \frac{\partial n_{\uparrow}}{\partial \rho_{\alpha \beta}}+\frac{\delta E_{\mathrm{xc}}\left[n_{\uparrow}, n_{\downarrow}\right]}{\delta n_{\downarrow}} \frac{\partial n_{\downarrow}}{\partial \rho_{\alpha \beta}} .
$$

The advantage is that we can find approximations for $\delta E_{\mathrm{xc}} / \delta n_{\sigma}$ by calculating the ground-state energy of a homogeneous system of spin densities $n_{\uparrow}$ and $n_{\downarrow}$, respectively. The price we pay is the derivatives $\partial n_{\uparrow} / \partial \rho_{\alpha \beta}$ and $\partial n_{\downarrow} / \partial \rho_{\alpha \beta}$, which introduce the local spin rotation angles $\theta(\mathbf{r})$ and $\varphi(\mathbf{r})$. Note that this method involves a change of variables in the exchange-correlation energy, and not a local 
rotation of the spinors. Such a rotation would lead to complicated terms in the kinetic energy, similar to those encountered in a field theory with local gauge transformations.

To actually perform the change of variables, we calculate at each point $\mathbf{r}$ an $S U(2)$ matrix $\mathbf{U}(\mathbf{r})$ that locally diagonalizes $\rho_{\sigma, \sigma^{\prime}}(\mathbf{r})$ :

$$
\sum_{\sigma, \sigma^{\prime}} U_{\alpha, \sigma}(\mathbf{r}) \rho_{\sigma, \sigma^{\prime}}(\mathbf{r}) U_{\sigma^{\prime}, \beta}^{*}(\mathbf{r})=\delta_{\alpha, \beta} n_{\alpha}(\mathbf{r}),
$$

with $n_{\alpha}(\mathbf{r})$ the eigenvalues of $\rho_{\sigma, \sigma^{\prime}}(\mathbf{r})$. We write $\mathbf{U}(\mathbf{r})$ in the standard form,

$$
\mathbf{U}(\mathbf{r})=\left(\begin{array}{cc}
e^{(i / 2) \varphi(\mathbf{r})} \cos \frac{\theta(\mathbf{r})}{2} & e^{-(i / 2) \varphi(\mathbf{r})} \sin \frac{\theta(\mathbf{r})}{2} \\
-e^{(i / 2) \varphi(\mathbf{r})} \sin \frac{\theta(\mathbf{r})}{2} & e^{-(i / 2) \varphi(\mathbf{r})} \cos \frac{\theta(\mathbf{r})}{2}
\end{array}\right),
$$

where $\varphi(\mathbf{r})$ and $\theta(\mathbf{r})$ are the local azimuthal and polar angles of the magnetization density relative to a fixed coordinate system. The requirement that $\mathbf{U}(\mathbf{r})$ diagonalizes $\rho_{\sigma, \sigma^{\prime}}(\mathbf{r})$ then yields

$$
\tan \varphi(\mathbf{r})=-\frac{\operatorname{Im} \rho_{\uparrow, \downarrow}(\mathbf{r})}{\operatorname{Re} \rho_{\uparrow, \downarrow}(\mathbf{r})}
$$

and

$$
\tan \theta(\mathbf{r})=\frac{2\left\{\left[\operatorname{Re} \rho_{\uparrow, \downarrow}(\mathbf{r})\right]^{2}+\left[\operatorname{Im} \rho_{\uparrow, \downarrow}(\mathbf{r})\right]^{2}\right\}^{1 / 2}}{\left[\rho_{\uparrow, \uparrow}(\mathbf{r})-\rho_{\downarrow, \downarrow}(\mathbf{r})\right]},
$$

with $\operatorname{Re}(z)$ and $\operatorname{Im}(z)$ denoting the real and imaginary parts of a complex number $z$. Equation (5) gives

$$
\begin{aligned}
n_{\uparrow}(\mathbf{r})= & \boldsymbol{\rho}_{\uparrow, \uparrow} \cos ^{2} \frac{\theta(\mathbf{r})}{2}+\frac{1}{2} \rho_{\uparrow, \downarrow}(\mathbf{r}) e^{i \varphi(\mathbf{r})} \sin \theta(\mathbf{r}) \\
& +\frac{1}{2} \rho_{\uparrow, \downarrow}^{*}(\mathbf{r}) e^{-i \varphi(\mathbf{r})} \sin \theta(\mathbf{r})+\rho_{\downarrow, \downarrow} \sin ^{2} \frac{\theta(\mathbf{r})}{2} \\
n_{\downarrow}(\mathbf{r})= & \rho_{\uparrow, \uparrow} \sin ^{2} \frac{\theta(\mathbf{r})}{2}-\frac{1}{2} \rho_{\uparrow, \downarrow}(\mathbf{r}) e^{i \varphi(\mathbf{r})} \sin \theta(\mathbf{r}) \\
& -\frac{1}{2} \rho_{\uparrow, \downarrow}^{*}(\mathbf{r}) e^{-i \varphi(\mathbf{r})} \sin \theta(\mathbf{r})+\rho_{\downarrow, \downarrow} \cos ^{2} \frac{\theta(\mathbf{r})}{2} .
\end{aligned}
$$

Equation (9) then gives us a representation in which $\rho_{\sigma, \sigma^{\prime}}(\mathbf{r})$ is locally diagonal, so that in the LDA we only need to know the exchange-correlation energy $E_{\mathrm{xc}}(\nu, \xi)$ as a function of total density and polarization, or, equivalently, $n_{\uparrow}$ and $n_{\downarrow}$. By using the $S U(2)$ transformation, and by expressing the single-particle density matrix in terms of occupied KS orbitals $\psi_{i, \sigma}(\mathbf{r})$ we can then write the KS equations as

$$
\begin{gathered}
T_{s} \psi_{i, \uparrow}(\mathbf{r})+v_{0}(\mathbf{r}) \psi_{i, \uparrow}(\mathbf{r})+\Delta v(\mathbf{r})\left[\cos \theta(\mathbf{r}) \psi_{i, \uparrow}(\mathbf{r})\right. \\
\left.+\sin \theta(\mathbf{r}) e^{i \varphi(\mathbf{r})} \psi_{i, \downarrow}(\mathbf{r})\right]=\epsilon_{i} \psi_{i, \uparrow} \\
T_{s} \psi_{i, \downarrow}(\mathbf{r})+v_{0}(\mathbf{r}) \psi_{i, \downarrow}(\mathbf{r})+\Delta v(\mathbf{r})\left[\sin \theta(\mathbf{r}) e^{-i \varphi(\mathbf{r})} \psi_{i, \uparrow}(\mathbf{r})\right. \\
\left.-\cos \theta(\mathbf{r}) \psi_{i, \downarrow}(\mathbf{r})\right]=\epsilon_{i} \psi_{i, \downarrow},
\end{gathered}
$$

for each single-particle spinors $\psi_{i}(\mathbf{r})=\left[\psi_{i, \uparrow}(\mathbf{r}), \psi_{i, \downarrow}(\mathbf{r})\right]$. Here,

$$
v_{0}(\mathbf{r})=v_{\mathrm{ext}}(\mathbf{r})+v_{\mathrm{H}}(\mathbf{r})+\frac{1}{2}\left[v_{\mathrm{xc}, \uparrow}(\mathbf{r})+v_{\mathrm{xc}, \downarrow}(\mathbf{r})\right],
$$

and

$$
\Delta v(\mathbf{r})=\frac{1}{2}\left[v_{\mathrm{xc}, \uparrow}(\mathbf{r})-v_{\mathrm{xc}, \downarrow}(\mathbf{r})\right],
$$

with

$$
v_{\mathrm{xc}, \sigma}=\frac{\delta E_{\mathrm{xc}}\left[n_{\uparrow}(\mathbf{r}), n_{\downarrow}(\mathbf{r})\right]}{\delta n_{\sigma}(\mathbf{r})} .
$$

In the KS equations (10) there is a coupling between up- and down-spin components, so that in general the KS orbitals are now two-component spinors with both up- and down-spin components. Note that in an angular momentum representation Eqs. (10) couple $z$ components of orbital angular momentum $L_{z}+m \hbar$, with $m$ an integer, and spin angular momentum $S_{z}=\frac{1}{2} \hbar$ to $\left(L_{z}, S_{z}=-\frac{1}{2} \hbar\right)$. This coupling will also provide the mechanism for spin-charge textures in QHE systems in the same way as the Hartree-Fock equations by Fertig et al. do. ${ }^{2}$ In the LDA, we write

$$
E_{\mathrm{xc}}=\int d^{2} r n(\mathbf{r}) \epsilon_{\mathrm{xc}}[\nu(\mathbf{r}), \xi(\mathbf{r})],
$$

where $\epsilon_{\mathrm{xc}}[\nu, \xi]$ is the exchange-correlation energy per particle in an infinite, homogeneous system of filling factor $\nu$ and polarization $\xi=\left(\nu_{\uparrow}-\nu_{\downarrow}\right) / n$, and $\nu(\mathbf{r})=2 \pi l_{B}^{2} n(\mathbf{r})$ is the density expressed as local filling factor. Then

$$
\begin{aligned}
& v_{\mathrm{xc}, \uparrow}(\mathbf{r})=\left[\frac{\partial}{\partial \nu}+\frac{1}{\nu}(1-\xi) \frac{\partial}{\partial \xi}\right]\left[\nu \epsilon_{\mathrm{xc}}(\nu, \xi)\right], \\
& v_{\mathrm{xc}, \downarrow}(\mathbf{r})=\left[\frac{\partial}{\partial \nu}-\frac{1}{\nu}(1+\xi) \frac{\partial}{\partial \xi}\right]\left[\nu \epsilon_{\mathrm{xc}}(\nu, \xi)\right],
\end{aligned}
$$

with the derivatives evaluated at $\nu=\nu(\mathbf{r})$ and $\xi=\xi(\mathbf{r})$, so that

$$
\begin{gathered}
v_{0}(\mathbf{r})=v_{\mathrm{ext}}(\mathbf{r})+v_{\mathrm{H}}(\mathbf{r})+\frac{\partial}{\partial \nu}\left[\nu \epsilon_{\mathrm{xc}}(\nu, \xi)\right]-\xi \frac{\partial}{\partial \xi} \epsilon_{\mathrm{xc}}(\nu, \xi), \\
\Delta v(\mathbf{r})=\frac{\partial}{\partial \xi} \epsilon_{\mathrm{xc}}(\nu, \xi) .
\end{gathered}
$$

Equations (10) with $v_{0}(\mathbf{r})$ and $\Delta v(\mathbf{r})$ given by Eq. (16) are the KS equations that result from the approach by Kübler et $a l .{ }^{25}$ and Sticht, Höck, and Kübler. ${ }^{22}$ Here they are written in a form appropriate for the QHE. We now make the extension to an ensemble DFT by introducing occupation numbers $f_{i}$ for each spinor $i$, and by taking

$$
\rho_{\sigma, \sigma^{\prime}}(\mathbf{r})=\sum_{i} f_{i} \psi_{i}^{\dagger}(\mathbf{r}) \hat{S}_{\sigma^{\dagger}}^{\dagger} \hat{S}_{\sigma^{\prime}} \psi_{i}(\mathbf{r}),
$$

where $\hat{S}_{\sigma}$ projects out the $\sigma$ component of the spinor $\psi_{i}(\mathbf{r})$. In ordinary DFT the occupancies $f_{i}$ are zero or one. In our ensemble DFT calculations, we obtain fractional occupation numbers using a method of running averages described in our earlier work. ${ }^{14,15}$

\section{FORMAL RESULTS AND NUMERICAL APPROXIMATIONS}

The usefulness of the LDA equations (10), (16), and (17) ultimately depends on the availability of good approximations for $\epsilon_{\mathrm{xc}}(\nu, \xi)$, the exchange-correlation energy per particle of a homogeneous system of filling factor $\nu$ and polar- 
ization $\xi$. We have previously ${ }^{16}$ described our first attempt at constructing an approximate energy surface for the QHE. In this section, we will describe in some detail our work to improve on that approximation. In particular, we have extended the exchange-correlation energy to the range $\nu>1$ and also better incorporated electron-hole symmetry.

We start, as in our earlier work, ${ }^{16}$ by approximating $\epsilon_{\mathrm{xc}}(\nu, \xi)$ as

$$
\epsilon_{\mathrm{xc}}(\nu, \xi)=\epsilon_{\mathrm{xc}}^{\mathrm{s}}(\nu)+\delta \epsilon_{\mathrm{xc}}(\nu) f(\xi)+\epsilon^{\mathrm{C}}(\nu) .
$$

Here, $\epsilon_{\mathrm{xc}}^{\mathrm{s}}(\nu)$ is a smooth interpolation formula for the ground state energy of polarized QHE systems, $\epsilon^{\mathrm{C}}(\nu)$ gives the cusps at the $\mathrm{FQHE}$ fractions, $f(\xi)$ is an interpolation formula obtained from considering only exchange in two dimensions,

$$
f(\xi)=\frac{(1+\xi)^{3 / 2}+(1-\xi)^{3 / 2}-2 \sqrt{2}}{2-2 \sqrt{2}},
$$

and $\delta \epsilon_{\mathrm{xc}}(\nu)$ is the difference in energy between the fully polarized and the completely unpolarized $(\xi=0)$ system at filling factor $\nu$. Some values for this latter quantity can be obtained from the literature, ${ }^{28-30}$ and the value for $\delta \epsilon_{\mathrm{xc}}(\nu$ $=1$ ) will be fixed later in this section using the spin stiffness. We then use a spline fit to tie all these values of $\delta_{\mathrm{xc}}(\nu)$ together to a continuous function. We found earlier ${ }^{16}$ that the interpolation formula for $\epsilon_{\mathrm{xc}}^{\mathrm{s}}(\nu)$ given by Fano and Ortolani ${ }^{31}$ together with our approximation for $\epsilon^{\mathrm{C}}(\nu)$ gave excellent agreement between our DFT approach and numerical diagonalizations ${ }^{32}$ for small spin-polarized systems. However, this interpolation formula was given only for $\nu$ $\leqslant 1$. To extend it to $\nu>1$, we have performed extensive numerical diagonalizations for toroidal spin-polarized systems of eight, nine, ten, and eleven particles in the two lowest Landau levels. ${ }^{33}$ The data obtained from these calculations reveal a cusp in the exchange-correlation energy at $\nu=1$. This cusp is due to the fact that the exchange-correlation energy per particle added to the second Landau level, after the lowest Landau level has filled up, is different from the exchange-correlation energy per particle for the filled lowest Landau level. We have confirmed this with analytical calculations (below), and constructed a simple analytical model to fit the numerical data. Figure 1 depicts the data from the numerical diagonalizations and the analytical fit.

We now briefly present the analytical calculation of the cusp in the exchange-correlation energy at $\nu=1$ for spinpolarized systems. We will do the calculation in a truncated Hilbert space, and take the state at $\nu=1$ to be a Slater determinant of the lowest Landau level single-particle wave functions. This is the exact ground state of the system restricted to the lowest Landau level, and the interaction energy is $E_{0}$ $=N_{\mathrm{el}} \epsilon_{\mathrm{xc}}(\nu=1, \xi=1)=-N_{\mathrm{el}} \sqrt{(\pi / 8)}\left(e^{2} / \epsilon_{0} l_{B}\right)$, where $N_{\mathrm{el}}$ is the number of electrons. For $N_{\mathrm{el}}+1$ particle we consider only states with $N_{\text {el }}$ particles occupying a filled lowest Landau level plus one particle in the $n=1$ Landau level. The lowest-energy state consisting of $N_{\mathrm{el}}+1$ particles is then a linear combination of these degenerate Slater determinants with a uniform density. The direct (Hartree) energy of this state is canceled by a uniform positive background charge density. We can calculate the exact energy of the lowestlying state (exact in the reduced Hilbert space used here) by considering the exchange interaction between a single par-

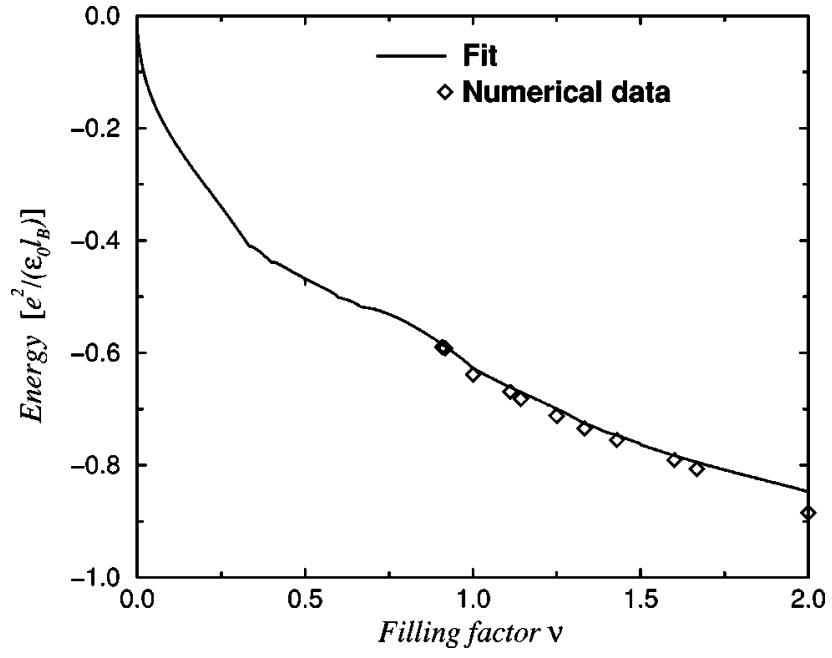

FIG. 1. Exchange-correlation energy vs filling factor for a spinpolarized QHE system. The diamonds are results from numerical diagonalizations in the two lowest Landau levels, and the solid line for $\nu>1$ is our analytical fit, Eq. (25).

ticle in Landau level $n=1$ at momentum $k_{0}=0$ and a particle of momentum $k$ in the lowest Landau level $n=0$. This energy is (using the Landau gauge)

$$
\begin{aligned}
\epsilon_{1}(k)= & -\iint d^{2} r d^{2} r^{\prime} \frac{1}{L_{y}^{2} \pi l_{B}^{2}} e^{-\left(1 / 2 l_{B}^{2}\right)\left(x-x_{k}\right)^{2}} e^{-i k y} \\
& \times e^{-\left(1 / 2 l_{B}^{2}\right) x^{\prime 2}} \frac{H_{1}\left(x^{\prime} / l_{B}\right)}{\sqrt{2}} V\left(\mathbf{r}-\mathbf{r}^{\prime}\right) \frac{H_{1}\left(x / l_{B}\right)}{\sqrt{2}} \\
& \times e^{-\left(1 / 2 l_{B}^{2}\right) x^{2}} e^{i k y^{\prime}} e^{-\left(1 / 2 l_{B}^{2}\right)\left(x^{\prime}-x_{k}\right)^{2}}
\end{aligned}
$$

Here, $L_{y}$ is the length of the system (taken to infinity at the end of the calculation), $H_{n}$ is the $n$th Hermite polynomial, $V(r)=e^{2} /\left(\epsilon_{0}\left|\mathbf{r}-\mathbf{r}^{\prime}\right|\right)$ the Coulomb interaction, and $x_{k}=l_{B}^{2} k$ is the centerpoint of the harmonic oscillator of momentum $k$. The integrals in Eq. (20) can be evaluated to give the result

$$
\epsilon_{1}(k)=-\frac{e^{2}}{2 L_{y} \epsilon_{0}} e^{-k^{2} l_{B}^{2} / 4} \frac{k^{2} l_{B}^{2}}{4}\left[K_{0}\left(k^{2} l_{B}^{2} / 4\right)+K_{1}\left(k^{2} l_{B}^{2} / 4\right)\right],
$$

where $K_{n}$ is the modified Bessel function of order $n$. We then finally integrate over all states $k$ in the lowest Landau level and obtain

$$
\epsilon_{1}=-\frac{1}{2} \sqrt{\frac{\pi}{8}} \frac{e^{2}}{\epsilon l_{B}}
$$

The cusp in ground-state energy at $\nu=1$ comes from the fact that the exchange energy of adding one particle to a system with a full lowest Landau level $\epsilon_{1}$ is different from the exchange energy per particle in the lowest Landau level. The cusp gives rise to a discontinuity in the chemical potential at $\nu=1$, which we need to evaluate. The chemical potential at $\nu=1^{-}$is $-\sqrt{\pi / 2} e^{2} /\left(\epsilon_{0} l_{B}\right)$, and the chemical potential at $\nu$ $=1^{+}$is 


$$
\begin{aligned}
\mu\left(\nu=1^{+}\right)= & \left.\frac{\partial E}{\partial N_{\mathrm{el}}}\right|_{\nu=1^{+}}=E\left(N_{\mathrm{el}}+1\right)-E\left(N_{\mathrm{el}}\right) \\
= & {\left[N_{\mathrm{el}} \epsilon_{\mathrm{xc}}(\nu=1, \xi=1)+\epsilon_{1}\right] } \\
& -N_{\mathrm{el}} \epsilon_{\mathrm{xc}}(\nu=1, \xi=1) \\
= & \epsilon_{1}=-\frac{1}{2} \sqrt{\frac{\pi}{8}} \frac{e^{2}}{\epsilon_{0} l_{B}} .
\end{aligned}
$$

Therefore, the discontinuity in chemical potential at $\nu=1, \xi$ $=1$ is

$$
\Delta \mu=\frac{3}{2} \sqrt{\frac{\pi}{8}} \frac{e^{2}}{\epsilon_{0} l_{B}} .
$$

Since the exchange-correlation potential for a polarized system is $V_{\mathrm{xc}}(\nu)=\partial\left[\nu \epsilon_{\mathrm{xc}}(\nu, \xi=1)\right] /(\partial \nu)=\mu(\nu)$ this discontinuity also appears in the exchange-correlation potential at $\nu=1$.

In order to construct an exchange-correlation energy surface $\epsilon_{\mathrm{xc}}(\nu, \xi)$ that gives a workable approximation for 0 $\leqslant \nu \leqslant 2$ and $0 \leqslant \xi \leqslant 1$ we first construct an analytic approximation for the exchange-correlation energy at $\nu>1$ and $\xi$ $=1$. We use a simple model in which we write

$$
\epsilon_{\mathrm{xc}}(\nu>1, \xi=1)=a \frac{\nu-1}{\nu} \epsilon_{\mathrm{xc}}(\nu-1, \xi=1)+\frac{c}{\nu}+b .
$$

This model is motivated by the fact that, as a first approximation, the interaction energy of a system with a full lowest Landau level and $N_{1}$ particles in the $n=1$ Landau level is approximately equal to the interaction energy of the full lowest Landau level plus the interaction energy of $N_{1}$ electrons in the lowest Landau level. The constants $b$ and $c$ then fix the slope and value of the exchange-correlation energy at $\nu$ $=1^{+}$and $\xi=1$, and $a$ is used to adjust this model to the numerical data. Fixing slope and value gives

$$
b=\frac{3}{2} \epsilon_{\mathrm{xc}}(\nu=1, \xi=1)
$$

and

$$
c=-\frac{1}{2} \epsilon_{\mathrm{xc}}(\nu=1, \xi=1),
$$

respectively. A good fit to the numerical data is given by $a$ $=2$.

Finally, we consider the exhange-correlation energy at $\xi$ $=0$. When the system is restricted to the lowest Landau level, particle-hole symmetry yields

$$
\begin{aligned}
\nu \epsilon_{\mathrm{xc}}(\nu, \xi)- & \nu \epsilon_{\mathrm{xc}}(\nu=1, \xi=1) \\
= & (2-\nu) \epsilon_{\mathrm{xc}}\left[2-\nu,\left(\nu_{\uparrow}-\nu_{\downarrow}\right) /(2-\nu)\right] \\
& -(2-\nu) \epsilon_{\mathrm{xc}}(\nu=1, \xi=1),
\end{aligned}
$$

where

$$
\nu=\nu_{\uparrow}+\nu_{\downarrow}>1
$$

and

$$
\xi=\frac{\nu_{\uparrow}-\nu_{\downarrow}}{\nu_{\uparrow}+\nu_{\downarrow}} .
$$

When the restriction of the system to the lowest Landau level is lifted, this symmetry is no longer exact because of interLandau-level quantum fluctuations. However, we assume that it is only slightly violated, and construct $\delta \epsilon_{\mathrm{xc}}(\nu)$ so that $\epsilon_{\mathrm{xc}}(\nu, \xi)$ respects this symmetry at $\xi=0$. Using the form Eq. (18) and Eq. (28) at $\xi=0$ and $\nu>1$ then yields

$$
\delta \epsilon_{\mathrm{xc}}(\nu)=\frac{[2-\nu]\left[\epsilon_{\mathrm{xc}}^{\mathrm{s}}(2-\nu)+\epsilon^{\mathrm{C}}(2-\nu)+\delta \epsilon_{\mathrm{xc}}(2-\nu)\right]-2(1-\nu) \epsilon_{\mathrm{xc}}(\nu=1, \xi=1)}{\nu}-\epsilon_{\mathrm{xc}}^{\mathrm{s}}(\nu)-\epsilon^{\mathrm{C}}(\nu)
$$

for $\nu>1$. Furthermore, at $\xi=0$ the exchange-correlation energy per particle has a continuous derivative at $\nu=1$, which gives

$$
\left.\frac{d\left(\delta \epsilon_{\mathrm{xc}}(\nu)\right)}{d \nu}\right|_{\nu=1}=-\epsilon_{\mathrm{xc}}(\nu=1, \xi=1)-\delta \epsilon_{\mathrm{xc}}(\nu=1) .
$$

Equations (18), (19), (25), (31), and (32), together with the data points for $\delta \epsilon_{\mathrm{xc}}(\nu)$ for $\nu \leqslant 1$, then define our exchangecorrelation energy surface. A F90 subroutine package that evaluates the exchange-correlation energy and the exchangecorrelation potentials for given $\left(\nu_{\uparrow}, \nu_{\downarrow}\right)$ is available from the authors. In this package, used in our calculations, we dropped the terms $\epsilon^{\mathrm{C}}$ for $\nu>1$. This is of no consequence in our calculations presented here in which the total filling factor was never much greater than unity.

To conclude this section, we show that the LDA does indeed give a spin stiffness; requiring this stiffness to be correct helps constrain $\delta \epsilon_{\mathrm{xc}}(\nu=1)$. We start by considering the total exchange-correlation energy of the system in the LDA,

$$
E_{\mathrm{xc}}=\int n(\mathbf{r}) \epsilon_{\mathrm{xc}}[\nu(\mathbf{r}), \xi(\mathbf{r})] d^{2} r
$$

where $\nu(\mathbf{r})$ and $\xi(\mathbf{r})$ are obtained from the local eigenvalues $n_{\uparrow}(\mathbf{r})$ and $n_{\downarrow}(\mathbf{r})$ of the single-particle density matrix. ${ }^{22} \mathrm{We}$ calculate in the LDA the change in exchange-correlation energy of an initially infinite, homogeneous, fully polarized system at $\nu=1$ in response to a gentle spin twist. The applied spin twist changes the local eigenvalues $n_{\sigma}(\mathbf{r})$ of the single-particle density matrix, and thus $\nu(\mathbf{r})$ and $\xi(\mathbf{r})$. For systems confined to the lowest Landau level, a spatially varying spin twist necessarily results in $\xi(\mathbf{r})<1 .{ }^{34}$ In the local-density approximation used here this produces a cost in exchange-correlation energy that, as we show here, causes the spin stiffness. We write

$$
\nu(\mathbf{r})=1+\delta \nu(\mathbf{r}), \quad \xi(\mathbf{r})=1+\delta \xi(\mathbf{r})
$$


The change in exhange-correlation energy is then [with $\left.n(\mathbf{r})=\nu(\mathbf{r}) /\left(2 \pi l_{B}^{2}\right)\right]$

$$
\begin{aligned}
\Delta E_{\mathrm{xc}}= & \int\left[\frac{1}{2 \pi}+\delta n(\mathbf{r})\right] \epsilon_{\mathrm{xc}}[1+\delta \nu(\mathbf{r}), 1+\delta \xi(\mathbf{r})] d^{2} r \\
& -\frac{1}{2 \pi} \int \epsilon_{\mathrm{xc}}(1,1) d^{2} r .
\end{aligned}
$$

From the work of Moon et al., ${ }^{35}$ we know that the density variation is of second order in the gradient of the spinrotation angle. Furthermore, general symmetry considerations give

$$
\delta \xi(\mathbf{r}) \propto[\nabla \boldsymbol{\Omega}(\mathbf{r})]^{2},
$$

where $\boldsymbol{\Omega}(\mathbf{r})=\hat{z} \times \mathbf{m}(\mathbf{r})$ is the angle through which the spin density is rotated, and $\mathbf{m}(\mathbf{r})$ is a unit vector parallel to the local spin density. We then expand $\Delta E_{\mathrm{xc}}$ in powers of $|\nabla \Omega|$, and obtain to second order in $|\nabla \Omega|$

$$
\begin{aligned}
\Delta E_{\mathrm{xc}}= & \int \delta n(\mathbf{r}) \epsilon_{\mathrm{xc}}(1,1) d^{2} r \\
& +\left.\frac{1}{2 \pi} \int \delta \nu(\mathbf{r}) \frac{\partial \epsilon_{\mathrm{xc}}}{\partial \nu}\right|_{\nu=1, \xi=1} d^{2} r \\
& +\left.\frac{1}{2 \pi} \int \delta \xi(\mathbf{r}) \frac{\partial \epsilon_{\mathrm{xc}}}{\partial \xi}\right|_{\nu=1, \xi=1} d^{2} r
\end{aligned}
$$

The point here is that an LDA in $\nu(\mathbf{r})$ and $\xi(\mathbf{r})$ contains spatially varying polarization $\xi(\mathbf{r})$, and this corresponds to gradients in the magnetization density according to Eq. (36). The first two terms on the left-hand side describe a change in $E_{\mathrm{xc}}$ due a change in the density. For the spin twist we are considering $\delta n(\mathbf{r})$ [and $\delta \nu(\mathbf{r})$ ] integrates to zero since no net charge is added to the system. The last term in Eq. (37) describes the change in $E_{\mathrm{xc}}$ due to a change in the polarization. This term gives the spin stiffness. In general, the spin stiffness $\rho_{s}$ is defined by the leading term in an expansion of the energy in gradients of the magnetization angle $\boldsymbol{\Omega}$ :

$$
E_{s}=\frac{1}{2} \rho_{s} \int[\nabla \mathbf{\Omega}(\mathbf{r})]^{2} d^{2} r
$$

We obtain from Eqs. (36) and (37)

$$
\left.\Delta E_{\mathrm{xc}} \propto \frac{1}{2 \pi} \frac{\partial \epsilon_{\mathrm{xc}}}{\partial \xi}\right|_{\nu=1, \xi=1} \int[\nabla \boldsymbol{\Omega}(\mathbf{r})]^{2} d^{2} r .
$$

By comparing Eqs. (38) and (39) we see that $\rho_{s}$ $\propto\left(\partial \epsilon_{\mathrm{xc}} / \partial \xi\right)$, and it remains to work out the constant of proportionality. To this end, we consider a system of spinpolarized electrons confined to the lowest Landau level. We use the Landau gauge $\mathbf{A}(\mathbf{r})=(0, B x, 0)$ in which the singleparticle basis functions are

$$
\psi_{k}(x, y)=e^{i k y} \phi_{k}(x)=\frac{e^{i k y} e^{-\left(1 / 2 l_{B}^{2}\right)\left(x-x_{k}\right)^{2}}}{\sqrt{L_{y}} \sqrt{\sqrt{\pi} l_{B}}},
$$

where

$$
x_{k}=l_{B}^{2} k, \quad k=2 \pi n / L_{y}, \quad n=0, \pm 1, \pm 2, \ldots .
$$

In the initial state, all single-particle spinors are

$$
\left(\begin{array}{c}
\psi_{k}(x, y) \\
0
\end{array}\right) \text {. }
$$

We take the spinors of the spin-rotated state to be

$$
\left(\begin{array}{c}
\cos \frac{\theta_{k}}{2} \psi_{k}(x, y) \\
\sin \frac{\theta_{k}}{2} \psi_{k-\Delta k}(x, y)
\end{array}\right)
$$

where $\Delta k=2 \pi \Delta n / L_{y}$, with $\Delta n$ a fixed integer. This state is the rectangular analogue of a rotationally symmetric skyrmion. In order to calculate the exchange-correlation energy of this state within the LDA, we then need to find the eigenvalues of the single-particle density matrix

$$
\rho_{\sigma, \sigma^{\prime}}(\mathbf{r})=\left(\begin{array}{cc}
\sum_{k} \cos ^{2} \frac{\theta_{k}}{2}\left|\psi_{k}(x, y)\right|^{2} & \sum_{k} \sin \frac{\theta_{k}}{2} \cos \frac{\theta_{k}}{2} \psi_{k}(x, y) \psi_{k-\Delta k}^{*}(x, y) \\
\sum_{k} \sin \frac{\theta_{k}}{2} \cos \frac{\theta_{k}}{2} \psi_{k}^{*}(x, y) \psi_{k-\Delta k}(x, y) & \sum_{k} \sin ^{2} \frac{\theta_{k}}{2}\left|\psi_{k-\Delta k}(x, y)\right|^{2}
\end{array}\right) .
$$

The eigenvalues are then readily obtained as

$$
\begin{aligned}
n_{\uparrow, \downarrow}(x)= & {\left[\sum_{k} \cos ^{2} \frac{\theta_{k}}{2} \phi_{k}^{2}(x)+\sum_{k} \sin ^{2} \frac{\theta_{k}}{2} \phi_{k-\Delta k}^{2}\right] } \\
& \pm \frac{1}{2}\left\{\left[\sum_{k} \cos ^{2} \frac{\theta_{k}}{2} \phi_{k}^{2}(x)-\sum_{k} \sin ^{2} \frac{\theta_{k}}{2} \phi_{k-\Delta k}^{2}\right]^{2}\right. \\
& \left.+\left[4 \sum_{k} \sin \frac{\theta_{k}}{2} \cos \frac{\theta_{k}}{2} \phi_{k}(x) \phi_{k-\Delta k}(x)\right]^{2}\right\}^{1 / 2}
\end{aligned}
$$

To continue, we will make use of the following results:

$$
\begin{gathered}
\sum_{k} \phi_{k}^{2}(x)=\frac{1}{2 \pi l_{B}^{2}}, \\
\sum_{k}\left(x-x_{k}\right)^{2} \phi_{k}^{2}(x)=\frac{1}{2 \pi l_{B}^{2}} \frac{l_{B}^{2}}{2}=\frac{1}{4 \pi}
\end{gathered}
$$




$$
\begin{aligned}
\phi_{k}(x) \phi_{k-\Delta k}(x) \approx & \phi_{k}(x)\left[\phi_{k}(x)-\Delta k \frac{\partial \phi_{k}(x)}{\partial k}\right. \\
& \left.+\frac{1}{2}(\Delta k)^{2} \frac{\partial^{2} \phi_{k}(x)}{\partial k^{2}}\right] .
\end{aligned}
$$

The second term on the right-hand side of Eq. (47) then vanishes when integrated over $k$. The third term contains

$$
(\Delta k)^{2}=\left(\frac{2 \pi}{L_{y}}\right)^{2}(\Delta n)^{2},
$$

which vanishes in the thermodynamic limit for fixed $\Delta n$ $\neq 0$. Therefore, we can approximate

$$
\sum_{k} \phi_{k}(x) \phi_{k-\Delta k}(x) \approx \sum_{k} \phi_{k}^{2}(x)=\frac{1}{2 \pi l_{B}^{2}} .
$$

Next, we assume that $\theta_{k}$ is slowly varying as a function of $k$. This means that in expressions such as

$$
\sum_{k} \cos ^{2} \frac{\theta_{k}}{2} \phi_{k}^{2}(x)=\frac{L_{y}}{2 \pi} \int d k \frac{\cos ^{2} \theta(k)}{2} \phi_{k}(x),
$$

where $\phi_{k}(x)$ as a function of $k$ is sharply peaked about $k$ $=x / l_{B}^{2}$, we can expand to the trigonometric function as a function of $k$ to second order in $k$ about $k=x / l_{B}^{2}$. When integrated over $k$, all first-order terms containing $d \theta / d k$ then vanish. Using Eq. (46) and $d^{2} \theta / d k^{2}=l_{B}^{4} d^{2} \theta /\left(d x^{2}\right)$ we obtain, after a little algebra,

$$
n_{\uparrow, \downarrow}=\frac{1}{2} \frac{1}{2 \pi l_{B}^{2}} \pm \frac{1}{2} \frac{1}{2 \pi l_{B}^{2}}\left[1-\frac{l_{B}^{2}}{4}\left(\frac{d \theta}{d x}\right)^{2}\right] .
$$

From the definition of the polarization $\xi(\mathbf{r})$ we then finally have

$$
\delta \xi(\mathbf{r})=-\frac{2 n_{\downarrow}(\mathbf{r})}{n_{\uparrow}(\mathbf{r})-n_{\downarrow}(\mathbf{r})}=-\frac{l_{B}^{2}}{4}\left(\frac{d \theta}{d x}\right)^{2} .
$$

Inserting this into Eq. (37) yields

$$
\Delta E_{\mathrm{xc}}=-\left.\frac{1}{4 \pi} \frac{\partial \epsilon_{\mathrm{xc}}}{\partial \xi}\right|_{\nu=1, \xi=1} \frac{1}{2} \int\left(\frac{d \theta}{d x}\right)^{2} d^{2} r .
$$

By comparing Eq. (38) and Eq. (53) we then obtain for the spin stiffness

$$
\rho_{s}=-\left.\frac{1}{4 \pi} \frac{\partial \epsilon_{\mathrm{xc}}}{\partial \xi}\right|_{\nu=1, \xi=1} .
$$

In fact, the calculation is readily generalized to an arbitrary spin-polarized filling factor $\nu_{0}$, so in general we have

$$
\rho_{s}=-\left.\frac{\nu_{0}}{4 \pi} \frac{\partial \epsilon_{\mathrm{xc}}(\nu, \xi)}{\partial \xi}\right|_{\nu=\nu_{0}, \xi=1} .
$$

In our approximation for the exchange-correlation energy, we can then fix $\delta \epsilon_{\mathrm{xc}}(\nu=1)$ (for which there is no known value) by requiring that the LDA spin stiffness Eq. (54) equals the known value ${ }^{35}$ for the spin stiffness at $\nu=1$,

$$
\rho_{s}=\frac{1}{16} \frac{1}{\sqrt{2 \pi}} \frac{e^{2}}{\epsilon_{0} l_{B}} .
$$

This yields

$$
\delta \epsilon_{\mathrm{xc}}(\nu=1)=\sqrt{\frac{\pi}{8}} \frac{2-\sqrt{2}}{3} \frac{e^{2}}{\epsilon_{0} l_{B}} \approx 0.1224 \frac{e^{2}}{\epsilon_{0} l_{B}} .
$$

As an indicator how good this value is for $\delta \epsilon_{\mathrm{xc}}(\nu=1)$, we consider the state at $\nu=1, \xi=0$ in an approximation in which the spin-up and spin-down particles are completely uncorrelated. In this case the energy per particle of that state is the same as the ground-state energy per particle at $\nu=\frac{1}{2}, \xi=1$, which is approximately ${ }^{31}-0.469 e^{2} /\left(\epsilon_{0} l_{B}\right)$. Neglecting the correlations between spin-up and spin-down particles should lead to an overestimate of the energy per particle. This approximation yields a difference in energy per particle between $\nu=1, \xi=0$ and $\nu=1, \xi=1$ of $\delta \epsilon_{\mathrm{xc}}(\nu=1)$ $=0.1577 e^{2} /\left(\epsilon_{0} l_{B}\right)$. In view of the fact that this is most likely an overestimate of the energy per particle at $\nu=1, \xi$ $=0$, we can conclude that the value $\delta \epsilon_{\mathrm{xc}}(\nu=1)$ $=0.1224 e^{2} /\left(\epsilon_{0} l_{B}\right)$ obtained from the spin stiffness is very reasonable. The value $\delta \epsilon_{\mathrm{xc}}(\nu=1)=0.1224 e^{2} /\left(\epsilon_{0} l_{B}\right)$ is the one we used in our calculations.

\section{EDGE RECONSTRUCTIONS OF QHE DOTS}

We have applied the ensemble spin DFT approach to circularly symmetric QHE dots and studied the edge reconstructions of such systems as a function of edge stiffness. We use a model in which the confining potential is supplied by a uniform positive background charge density. The edge is modeled by a "graded edge" in which the positive background charge density goes to zero linearly as a function of radial coordinate $r$ over a distance $w$. The total integrated positive charge is fixed and equal to the total electron charge; this then determines the radial distance at which the positive charge density starts to decrease. Even though concerns have been raised that this particular confinement is nongeneric ${ }^{12}$ it is the one that has been studied the most, and we chose it as a model confinement for comparisons with other work.

It is known from Hartree-Fock calculations ${ }^{10}$ of $\nu=1$ Hall bars with a similar graded edge that for a sharp edge ( $w$ small enough), the electron gas is completely polarized and its density falls to zero abruptly near the edge. However, as the edge confinement softens, the electron gas develops an instability to a spin-textured edge for smaller values of $\tilde{g}$ (the ratio of Zeeman to Coulomb energy), or a chargedensity wave ${ }^{11,12}$ for larger values of $\tilde{g}$, with the density modulated along the edge. Both instabilities break the translational invariance along the edge: in the spin-textured edge the spin density is modulated along the edge while the total charge density is constant; in the charge-density wave edge the spin density is constant along the edge while the total charge density is modulated. For Hall bars at bulk filling $\nu$ $=1 / 3$, an effective field calculation ${ }^{10}$ (here, Hartree-Fock calculations are obviously not applicable) also shows an instability to a spin-textured edge as the confinement is softened.

We would expect the analogous instabilities to occur for the circular dots. For stiff confinements, the electron density forms a so-called maximum density droplet (MDD), in which the electron gas is completely polarized with a filling factor that is unity in the bulk and that rapidly falls to zero at 
the edge (the MDD is the minimum angular momentum state for a spin-polarized system in the lowest Landau level). As the confinement is softened, the edge should develop a spintextured or charge-density wave instability. Indeed, our previous spin DFT calculations, ${ }^{16}$ in which the magnetization direction was constrained to be fixed parallel to the external magnetic field, revealed that the MDD becomes unstable towards the formation of a partially polarized edge as the confinement was softened. This gave a variational bound (within the LDA) showing that the spin-polarized edge is not the ground state when the confinement is soft. The phase boundaries of the MDD that we obtained were in good qualitative agreement with Hartree-Fock calculations for Hall bars, ${ }^{10}$ although the obtained values of $\tilde{g}$ at which the polarization was destroyed were much smaller than those from the Hartree-Fock calculation for Hall bars. We speculated that this difference is due to the different geometries or edge confinements. Our results were in rather good agreement with numerical diagonalizations using parabolic confinement. ${ }^{32}$ The calculations presented here support the argument that the differences were due to the different confinements used.

We have now extended our ensemble DFT calculations to include spin-textured edges both for the MDD and for $\nu$ $=1 / 3$ droplets. Our new results show that when the edge becomes partially polarized, a spin-textured edge has lower energy than one with constant direction of the spin density. In the calculations presented here, we have only considered states that do not break cylindrical symmetry of the charge density, and for which the azimuthal angle of the spin direction changes at most by $2 \pi$ along any simple closed path. This excludes charge-density-wave instabilities and spin textures with topological charge greater than $\pm e$, but imposing these symmetries simplifies the calculations a great deal. For example, the Hartree potential is easy to calculate for circularly symmetric charge densities, but considerably more tedious if that symmetry is broken. Nevertheless, the calculations we have performed satisfy the two most important criteria we wanted to establish: to demonstrate the usefulness of the spin ensemble DFT approach to general QHE fillings, and to establish through a variational bound (within the LDA) that for softer edges, the spin-polarized edge or an edge with constant spin quantization axis has higher energy than a spin-textured system in which the spin quantization axis is tumbling.

We start with the ensemble spin KS equations Eq. (10) with the potentials $v_{0}(\mathbf{r})$ and $\Delta v(\mathbf{r})$ given by Eqs. (16), and the single-particle density matrix found self-consistently using Eq. (17). We now make the simplifying assumption that the polar angle $\theta(\mathbf{r})$ of the spin density is a function of the radial coordinate alone, $\theta(\mathbf{r})=\theta(r)$, and that the azimuthal angle $\varphi(\mathbf{r})$ is of the form

$$
\varphi(\mathbf{r})=v \phi(\mathbf{r})
$$

where $v$ is an integer and $\phi(\mathbf{r})$ is the azimuthal angle of $\mathbf{r}$ in a planar polar coordinate system. In other words, we will restrict the modulation of the spin density along the edge to have a single Fourier component along the edge. With Eq. (58) inserted into the expressions Eq. (10) for $v_{0}(\mathbf{r})$ and $\Delta v(\mathbf{r})$ the spin-diagonal coupling conserves orbital angular momentum, while the spin off-diagonal coupling couples up- spin states $\psi_{m+v, \uparrow}$ with angular momentum $\hbar(m+v)$ to down-spin states $\psi_{m, \downarrow}$ with angular momentum $\hbar m$ :

$$
\int \cos \theta(r) \psi_{m, \downarrow}^{*}(\mathbf{r}) \Delta v(r) e^{-i v \phi} \psi_{m+v, \uparrow}(\mathbf{r}) d^{2} r .
$$

This coupling is of the same form as that in the Hartree-Fock equations studied first by Fertig et al. ${ }^{2}$ However, in the Hartree-Fock equations the off-diagonal coupling was provided by an exchange integral, while our Eq. (59) also includes correlation effects within the LDA. Since the spindiagonal coupling conserves angular momentum, the diagonal elements of the single-particle density matrix are circularly symmetric so that the total density is circularly symmetric. We would like to point out that lifting the restriction Eq. (58) allows for the possibility of breaking the circular symmetry of the charge density, which allows for chargedensity waves in addition to spin-charge structures.

We have solved the KS equations by expanding the spatial parts of each spinor $\psi_{i}(\mathbf{r})$ in the single-particle angular momentum basis functions

$$
\psi_{m, n}(\mathbf{r})=\frac{1}{\sqrt{2 \pi} l_{B}} \sqrt{\frac{n !}{(n+m) !}} L_{m}^{n}\left(\frac{r^{2}}{2 l_{B}}\right) e^{i m \phi},
$$

for the cylindrical gauge $\mathbf{A}(\mathbf{r})=\frac{1}{2} B(x \hat{\mathbf{y}}-y \hat{\mathbf{x}})$ with $L_{m}^{n}$ the associated Laguerre polynomials. We kept up to the $n=4$ Landau level in our calculations, and up to 120 angular momentum states (up to 1200 single-particle states). For the $\nu$ $=1$ system we performed calculations of 40 and 70 particles in a magnetic field of $3.5 \mathrm{~T}$ with the bare Lande factor $g^{*}$ varying from 0.1 to 1.5 ( $\tilde{g}$ then varied from about 0.002 to 0.036 , encompassing experimentally accessible values). The reason for keeping the magnetic field relatively low, but still at an experimentally realistic value, was to fully include the effects of Landau-level mixing typical in experimental system. We performed the calculations for $v=0, \pm 1$. The results can be summarized as follows: For small values of $w$, i.e., stiff confinement, the edge is spin polarized. As $w$ increases, the $v=0$ channel becomes partially polarized. However, at the same value of $w$, the $v=1$ channel attains a lower energy with a nontrivial spin-charge texture. For the system sizes studied here, the value of $w$ at which the instability occurred is $w \approx 7 l_{B}$. This is in quite good agreement with the HartreeFock calculations of Karlhede et al. ${ }^{10}$ For a semi-infinite Hall bar, they found the onset to a charge-spin textured edge occurring at about $w \approx 7 l_{B}-8 l_{B}$ in the range of $\tilde{g}$ from zero to about 0.03. As illustrations, Figs. 2 and 3 depict charge densities and spin rotation angles for $w=8 l_{B}$ and $g=1.00$. The $v=0$ channel (Fig. 2) has a small minority-spin density near the edge of the system, in qualitative agreement with our earlier calculations (the bump in density at the edge is characterisic of all confined QHE systems for which the confining potential is not macroscopically smooth). However, the $v=1$ channel (Fig. 3), which has lower energy, has a locally (nearly) polarized spin density everywhere and a nontrivial spin texture, with the spin rotation angle $\theta(r)$ rising from 0 to about $\pi / 2$ at the edge of the system. Note that for a bounded system there is no topological constraint on the spin rotation angle, as there is in an infinite system where the Pontryagin index has to be an integer. 


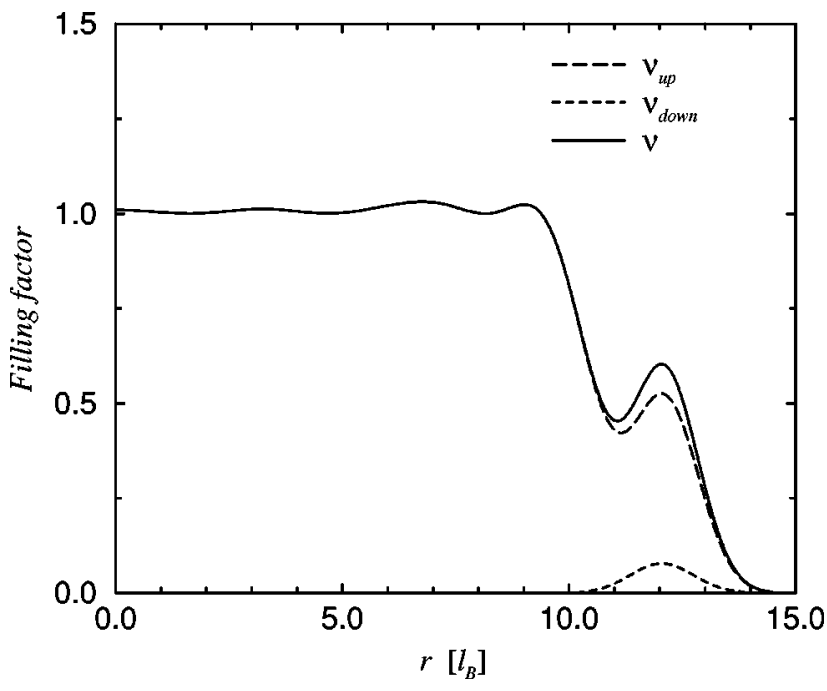

FIG. 2. Local filling factors $\nu_{\uparrow}(\mathbf{r}), \nu_{\downarrow}(\mathbf{r})$, and $\nu(\mathbf{r})=\nu_{\uparrow}(\mathbf{r})$ $+\nu_{\downarrow}(\mathbf{r})$ in the $v=0$ channel for a 70 -electron dot. The magneticfield strength is $3.5 \mathrm{~T}, g^{*}=1.0$, and the edge width is $w=8 l_{B}$. Here, the edge region is only partially polarized with a small minority-spin density.

We have also studied a FQHE droplet at bulk filling of $1 / 3$. While we are quite confident about our exchangecorrelation energy for spin-polarized FQHE systems, it is not clear to us how good it is for arbitrary polarizations in the FQHE regime. For example, if we use the published value obtained from four-electron numerical diagonalizations ${ }^{28}$ for $\delta \epsilon_{\mathrm{xc}}(\nu=1 / 3)$ of $0.0017 e^{2} /\left(\epsilon_{0} l_{B}\right)$, the spin-stiffness in our model is $1.155 \times 10^{-4} e^{2} /\left(\epsilon_{0} l_{B}\right)$, compared to the value of $9.23 \times 10^{-4} e^{2} /\left(\epsilon_{0} l_{B}\right)$ obtained from hypernetted-chain calculations. ${ }^{35}$ It is not clear if the discrepancy between these

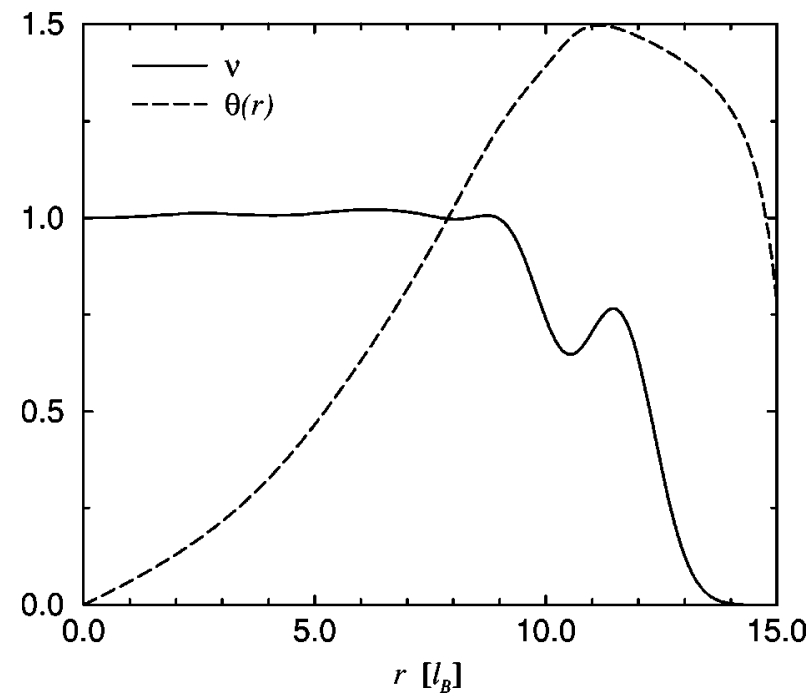

FIG. 3. The local total filling factor and spin rotation angle $\theta(r)$ for the $v=1$ channel of the same parameters as in Fig. 2. This channel has a lower energy than the $v=0$ channel, and is locally nearly completely polarized with a nontrivial spin texture. That is, at any point $r$ the spin density is nearly polarized, but the spin direction is changing with position. (In agreement with the general properties of the lowest Landau level, as described in the text, the polarization in fact deviates slightly from unity by an amount of order $(d \theta / d r)^{2}$. This is too small here to show up in the figures.)

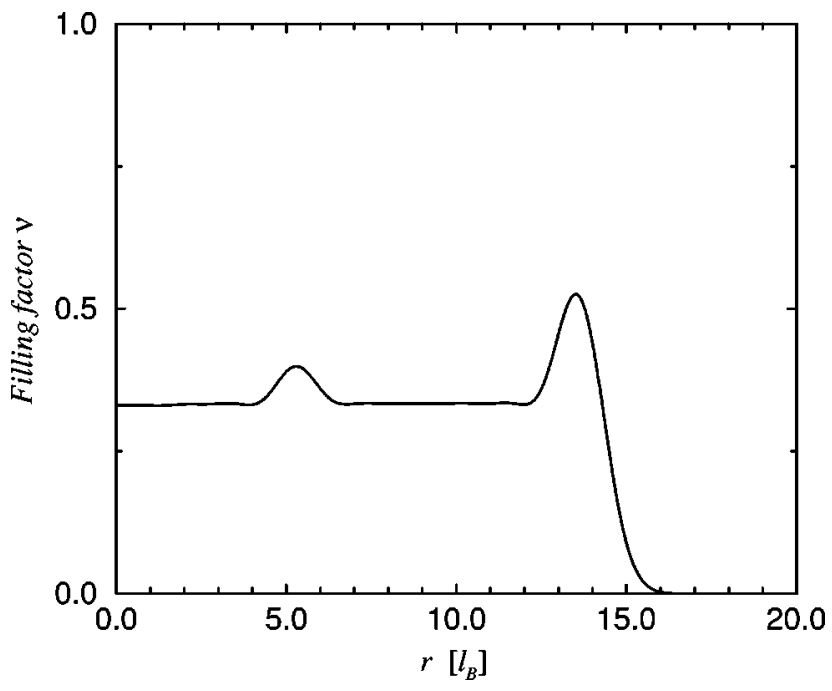

FIG. 4. The local filling factor for the $v=0$ channel of a 40electron dot in the FQHE regime. The external magnetic-field strength is $12 \mathrm{~T}, g^{*}=1.0$, and $w=4 l_{B}$. In this case, the electron system is polarized.

two is mostly due to finite-size effects in the numerical diagonalizations, or our model Eqs. (18) and (19) of the exchange-correlation energy. We have also performed numerical diagonalizations of six electrons at $\nu=1 / 3$. For six electrons, we obtain $\delta \epsilon_{\mathrm{xc}}(\nu=1 / 3)=0.00465 e^{2} /\left(\epsilon_{0} l_{B}\right)$-an increase of almost a factor of three from the four-electron result. This clearly shows that the energy per particle at total spin zero is much more sensitive to system size than the energy of the polarized ground state. Encouraged by our numerical results, we then fixed $\delta \epsilon_{\mathrm{xc}}(\nu=1 / 3)$ to $0.0136 e^{2} /\left(\epsilon_{0} l_{B}\right)$ in order to have a simple model that gives a spin stiffness in agreement with the hypernetted chain calculations. We performed the calculation with 40 particles and the four lowest Landau levels, and up to 170 single-particle angular momentum states, both for the choice of confinement discussed earlier and parabolic confinement.

Our calculations indicate that for a confinement provided by the positive background charge density, the system has an instability from a spin-polarized edge to a spin-textured edge at an edge width of $w \approx 4 l_{B}$. Again, this compares rather well with the results of Karlhede et al. ${ }^{10}$ Using effective-field theories, they found an instability to a spin-textured edge at approximately $w=3.0 l_{B}$ for $\tilde{g}=0.04$. Note that the effectivefield theory tends to underestimate the value of $w$ for which there is an onset to spin-textured edges. ${ }^{10}$ We also want to emphasize that in contrast to the effective-field (and HartreeFock) theory, our ensemble spin DFT is applicable to general inhomogeneous QHE system and includes the effects of Landau-level mixings. As an example of our results, we show in Figs. 4 and 5 charge densities and spin rotation angles for a system with an edge width of $w=4$ and Landé factor of $g^{*}=1.00$ in a magnetic field of $12 \mathrm{~T}(\tilde{g} \approx 0.04)$. The bump in total filling factor in the bulk of the $v=0$ channel (Fig. 4) occurs quite generically for FQHE droplets, both for the choice of confinement discussed here and for parabolic confinement. In the case here, the system tries to take advantage of correlation energy at $\nu=1 / 3$ and $\nu=2 / 5$ to as large an extent as possible as the edge is made wider. It does 


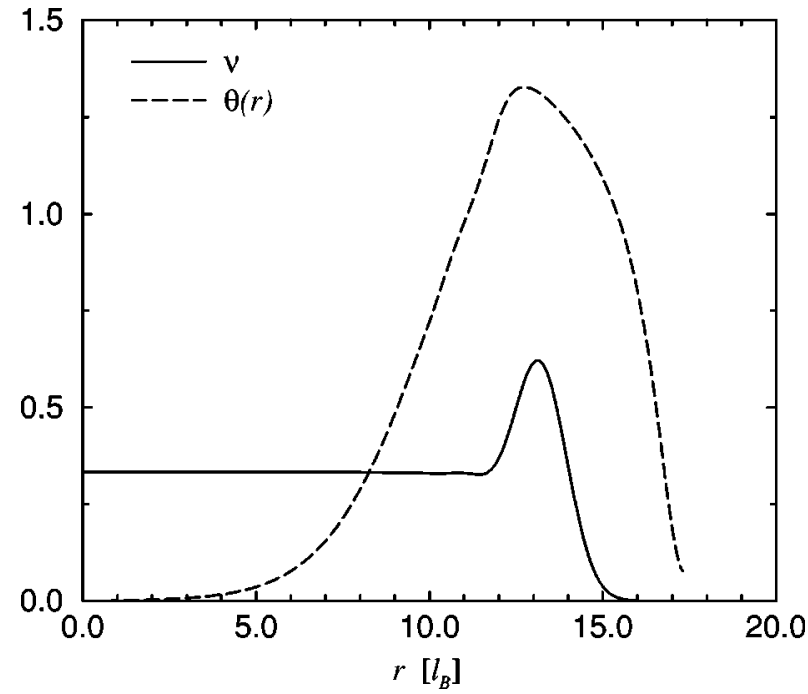

FIG. 5. The $v=1$ channel for the same system as in Fig. 4. This channel is also nearly completely polarized, but has a nontrivial spin texture and lower energy than the $v=0$ channel.

so by making the edge of the electron density sharper than that of the background charge, and making regions of $\nu$ $=1 / 3$ larger than what is needed to accomodate all electron charge. The residual electron charge is piled up in a bump reaching $\nu=2 / 5$. The $v=1$ channel, on the other hand, is locally nearly completely polarized with a spin rotation angle similar to the $\nu=1$ and $w=8$ system for $v=1$.

We have also performed calculations for parabolically confined systems in the FQHE regime. The results are in this case more difficult to interpret with more complicated structures in the electron density and spin textures. For example, we have found that for some values of the magnetic field the $v=0$ channel can be a $\nu=1 / 3$ droplet with a large bump in density at the edge, while the $v=1$ channel develops a hole with reversed spin density at the center and has lower energy for a small range of magnetic field.

\section{CONCLUSIONS AND SUMMARY}

We have here presented an ensemble spin DFT approach to general inhomogeneous QHE systems. This approach includes the spin degree of freedom, including noncollinear spins, as well as Landau-level mixing. On a formal level, we have demonstrated that our ensemble spin DFT in the LDA can give the correct spin stiffness at $\nu=1$. We have performed model calculations for circularly symmetric QHE dots in the integer and FQHE regime. These calculations show, in agreement with Hartree-Fock and effective-field calculations, ${ }^{10-12}$ that the polarized system develops an instability as the confinement is softened and that the spintextured edge attains a lower energy than the spin-polarized one. We have not included in our calculations the possibilities of charge-density waves, which may occur ${ }^{11,12}$ instead of the spin-textured edges at larger values of $\tilde{g}$. Preliminary calculations of parabolic dots show a surprisingly rich structure in spin and charge densities. This indicates that quantum dots in the FQHE regime is a rich subject yet to be fully explored.

We have spent a great deal of effort on improving our approximation for the exchange-correlation energy $\epsilon_{\mathrm{xc}}(\nu, \xi)$. At the present, we are confident that we have a very good approximation for spin-polarized systems, and a good approximation for aribitrary polarizations and $\nu \approx 1$. We are less confident about our exchange-correlation energy for arbitrary polarizations in the FQHE regime. Work needs still to be done to refine the exchange-correlation energy for general FQHE systems. However, we are confident that the approach itself is robust and accurate provided good approximations for the exchange-correlation energy exist.

\section{ACKNOWLEDGMENTS}

The authors are greatly indebted to Professor E.K.U. Gross for pointing out the work on noncollinear spins by Kübler and coworkers. We would also like to thank Professor Walter Kohn for stimulating and challenging comments on our ensemble DFT approach, and Professor A.H. MacDonald for being a general source of wisdom of the QHE to whom we can always turn for good comments and inspiration. O.H. and M.D.J. are grateful for support from the NSF through Grant No. DMR96-32141, and O.H. would also like to thank S. Östlund and M. Jonson at Chalmers University of Technology and Göteborg University for support and hospitality during a sabbatical leave where this work was started, and P. Hawrylak and C. Dharma-Wardana for a productive visit at the National Research Council.
*Present and permanent address: Seagate Technology, 7801 Computer Ave. South, Bloomington, MN 55435.

${ }^{1}$ S.L. Sondhi, A. Karlhede, S.A. Kivelson, and E.H. Rezayi, Phys. Rev. B 47, 16419 (1993); E.H. Rezayi, ibid. 36, 5454 (1987).

${ }^{2}$ H.A. Fertig, L. Brey, R. Cotê, and A.H. MacDonald, Phys. Rev. B 50, 11018 (1994).

${ }^{3}$ For an excellent review, see S.M. Girvin and A.H. MacDonald, in Novel Quantum Liquids in Low-Dimensional Semiconductor Structures, edited by S. Das Sarma and A. Pinczuk (Wiley, New York, 1995).

${ }^{4}$ T.H.R. Skyrme, Proc. R. Soc. London, Ser. A 262, 233 (1961); A.A. Belavin and A.M. Polyakov, JETP Lett. 22, 245 (1975).

${ }^{5}$ L. Brey, H.A. Fertig, R. Côté, and A.H. MacDonald, Phys. Rev. Lett. 75, 2562 (1995).

${ }^{6}$ R.K. Kamilla, X.G. Wu, and J.K. Jain, Solid State Commun. 99, 289 (1996)
${ }^{7}$ S.E. Barrett, G. Dabbagh, L.N. Pfeiffer, K. West, and R. Tycko, Phys. Rev. Lett. 74, 5112 (1995).

${ }^{8}$ A. Schmeller, J.P. Eisenstein, L.N. Pfeiffer, and K.W. West, Phys. Rev. Lett. 75, 4290 (1995).

${ }^{9}$ E.H. Aifer, B.B. Goldberg, and D.A. Broido, Phys. Rev. Lett. 76, 680 (1996).

${ }^{10}$ A. Karlhede, S.A. Kivelson, K. Lejnell, and S.I. Sondhi, Phys. Rev. Lett. 77, 2061 (1996).

${ }^{11}$ M. Franco and L. Brey, SISSA cond-mat/9702239 (unpublished).

${ }^{12}$ A. Karlhede and K. Lejnell, SISSA cond-mat/9709339 (unpublished).

${ }^{13}$ J.H. Oaknin, L. Martin-Moreno, and C. Tejedor, Phys. Rev. B 54, 16850 (1996).

${ }^{14}$ O. Heinonen, M.I. Lubin, and M.D. Johnson, Phys. Rev. Lett. 75, 4110 (1995). 
${ }^{15}$ O. Heinonen, M.I. Lubin, and M.D. Johnson, Int. J. Quantum Chem., Quantum Chem. Symp. 30, 231 (1996).

${ }^{16}$ M.I. Lubin, O. Heinonen, and M.D. Johnson, Phys. Rev. B 56, 10 373 (1997).

${ }^{17}$ U. von Barth and L. Hedin, J. Phys. C 5, 1629 (1972).

${ }^{18}$ P. Hohenberg and W. Kohn, Phys. Rev. 136, B864 (1964).

${ }^{19}$ W. Kohn and L.J. Sham, Phys. Rev. 140, A1133 (1965).

${ }^{20}$ For 2D electron gas, see B. Tanatar and D.M. Ceperley, Phys. Rev. B 39, 5005 (1989); Y. Kwon, D.M. Ceperley, and R.M. Martin, ibid. 48, 12037 (1993).

${ }^{21}$ For 3D electron gas, see, for example, J.P. Perdew and A. Zunger, Phys. Rev. B 23, 5048 (1981).

${ }^{22}$ J. Sticht, K.-H. Höck, and J. Kübler, J. Phys.: Condens. Matter 1, 8155 (1989).

${ }^{23}$ M. Uhl, L.M. Sandratskii, and J. Kübler, Phys. Rev. B 50, 291 (1994).

${ }^{24}$ L.M. Sandratskii and J. Kübler, Phys. Rev. Lett. 75, 946 (1995).

${ }^{25}$ J. Kübler, K.-H. Höck, J. Sticht, and A.R. Williams, J. Phys. F 18, 469 (1988).

${ }^{26}$ This approach was recently reviewed in L.M. Sandratskii, Adv. Phys. 47, 91 (1998).
${ }^{27}$ L.M. Sandratskii and J. Kübler, HCM Newsletter of the $\Psi_{k}$ Network 14, 42 (1996).

${ }^{28}$ F.C. Zhang, T. Chakraborty, Phys. Rev. B 30, 7320 (1984).

${ }^{29}$ P.A. Maksym, J. Phys.: Condens. Matter 1, L6299 (1989).

${ }^{30}$ See also Quantum Hall Effects, by T. Chakraborty and P. Pietiläinen (Springer, New York, 1995).

${ }^{31}$ G. Fano and F. Ortolani, Phys. Rev. B 37, 8179 (1988).

${ }^{32}$ S.-R. Eric Yang, A.H. MacDonald, and M.D. Johnson, Phys. Rev. Lett. 73, 3194 (1993).

${ }^{33}$ Our code is a FORTRAN 90 code based on an earlier code written by D. Yoshioka. We thank A.H. MacDonald for providing that code to us.

${ }^{34} \mathrm{~A}$ general proof of this assertion can be constructed using the operator algebra for projection onto the lowest Landau level (Ref. 35). More simply, one can readily show that applying a nonuniform spin twist to an initially polarized filled lowest Landau level, and then projecting into the lowest level, produces a reduced polarization $\xi(\mathbf{r})<1$. The calculation of spin stiffness in this paper shows the same point explicitly.

${ }^{35}$ K. Moon et al., Phys. Rev. B 51, 5138 (1995). 